\title{
ESTUDO PROSPECTIVO COMPARATIVO DE DUAS MODALIDADES DE POSICIONAMENTO DO SENSOR DE PHMETRIA ESOFÁGICA PROLONGADA: por manometria esofágica e pela viragem do $\mathrm{pH}$
}

\author{
Ary NASI ${ }^{1,3}$, Rita de Cássia FRARE², Jeovana F. BRANDÃ $0^{1,2}$, Ângela M. FALCÃ $0^{1,2}$, \\ Nelson H. MICHELSOHN ${ }^{3}$ e Daniel SIFRIM ${ }^{4}$
}

RESUMO - Racional - Por padronização aceita internacionalmente, posiciona-se o sensor distal de pHmetria esofágica a $5 \mathrm{~cm}$ acima da borda superior do esfíncter inferior do esôfago, localizado por manometria esofágica. Porém, vários autores sugerem técnicas alternativas de posicionamento que prescindem da manometria. Dentre essas, destaca-se a da viragem do $\mathrm{pH}$, tema este controverso pela sua duvidosa confiabilidade. Objetivo - Avaliar a adequação do posicionamento do sensor distal de pHmetria pela técnica de viragem do pH, considerandose a presença, o tipo e o grau de erro de posicionamento que tal técnica proporciona, e também estudar a influência da posição adotada pelo paciente durante a técnica da viragem. Métodos - Foram estudados de modo prospectivo, durante o período de 1 ano, 1.031 pacientes. Durante entrevista clínica, foram registrados os dados demográficos e as queixas clínicas apresentadas. Todos foram submetidos a manometria esofágica para localização do esfíncter inferior do esôfago e a técnica da viragem do $\mathrm{pH}$. A identificação do ponto de viragem foi realizada de dois modos distintos, caracterizando dois grupos de estudo: com o paciente sentado (grupo I - 450 pacientes) e com o paciente em decúbito dorsal horizontal (grupo II - 581 pacientes). Após a identificação do ponto de viragem, o sensor distal de pHmetria era posicionado na posição padronizada, baseada na localização manométrica do esfíncter. Registrava-se onde seria posicionado o sensor de $\mathrm{pH}$ se fosse adotada a técnica da viragem. Para avaliação da adequação do posicionamento, considerou-se que o erro é representado pela diferença (em centímetros) entre a localização padronizada (manométrica) e a localização que seria adotada caso fosse empregada a técnica da viragem. Considerou-se que o erro seria grosseiro se fosse maior que $2 \mathrm{~cm}$. Analisou-se também o tipo de erro mais freqüente (se acima ou abaixo da posição padronizada). Foram incluídos todos pacientes que aceitaram participar da pesquisa e excluídos os casos nos quais não se identificou acidificação intragástrica. Resultados - Se fosse adotada a técnica da viragem, haveria erro no posicionamento do sensor em 945 pacientes (91,6\%), portanto, o sensor seria posicionado na posição padronizada em apenas 86 (8,4\%) casos. Em relação à caracterização do grau de erro, haveria erro considerado grosseiro em 597 (63,2\%) pacientes. Em relação ao tipo de erro, o sensor seria posicionado abaixo do local padronizado em 857 (90,7\%) casos. Não houve diferença significante entre os dois grupos de estudo em relação a nenhum dos parâmetros analisados, indicando que a posição adotada pelo paciente durante a manobra da viragem não interfere no erro inerente à técnica. Conclusões - 1. O posicionamento do sensor distal de $\mathrm{pHmetria}$ pela técnica da viragem do $\mathrm{pH}$ não é confiável. 2. A técnica da viragem proporciona margem de erro expressiva. 3. O tipo de erro mais comum que tal modalidade técnica proporciona é o posicionamento mais distal do sensor, que pode superestimar a ocorrência de refluxo. 4. Não há influência da posição adotada pelo paciente durante a realização da técnica da viragem do $\mathrm{pH}$ na eficiência do método.

DESCRITORES - Monitoramento do pH esofágico. Refluxo gastroesofágico, diagnóstico. Esofagite péptica, diagnóstico. Esfíncter esofágico inferior. Estudo comparativo

\footnotetext{
Trabalho realizado no Hospital das Clínicas da Faculdade de Medicina da Universidade de São Paulo.

Laboratório de Investigação Funcional do Esôfago do Serviço de Cirurgia do Esôfago do Hospital das Clínicas da Faculdade de Medicina da Universidade de São Paulo - FMUSP; ${ }^{2}$ Curso de Pós-graduação, Departamento de Gastroenterologia da FMUSP; ${ }^{3}$ Setor de Motilidade Digestiva do Fleury: Medicina e Saúde; ${ }^{4}$ Centro de Pesquisa Gastroenterológica da Universidade de Leuven, Bélgica.
}

Correspondência: Dr. Ary Nasi - Rua Oscar Freire, 1702 - apt. 88 - 05409-011 - São Paulo, SP, E-mail: ary.nasi@fleury.com.br 


\section{INTRODUÇÃO}

A doença do refluxo gastroesofágico (DRGE), considerada uma das afecções digestivas de maior prevalência nos países ocidentais $^{(18,19,20)}$, tem sido bastante estudada. Merece destaque o grande número de publicações existentes na literatura médica sobre o tema. Contudo, ainda permanecem muitos temas controversos; um deles motivou a realização do presente estudo.

A pHmetria esofágica prolongada, introduzida na prática clínica na década de 70, propiciou grande incremento no conhecimento da doença. Por normatização aceita internacionalmente, posiciona-se o sensor de pHmetria a $5 \mathrm{~cm}$ acima da borda superior do esfíncter inferior do esôfago (EIE), para evitar que em casos de migração de parte da câmara gástrica para o tórax ou durante o encurtamento natural do esôfago que ocorre no processo de deglutição, haja contato do sensor com o conteúdo gástrico e conseqüente detecção de pseudorefluxos ${ }^{(3)}$. Com tal padronização, foram estabelecidos todos os parâmetros de normalidade do método ${ }^{(3,7,9)}$.

Considera-se que o melhor método de posicionamento do sensor de pHmetria seja a localização precisa do EIE por manometria esofágica ${ }^{(1,3,4,10,15,16,17)}$. Há, porém, propostas de técnicas alternativas que prescindem da realização da mesma; dentre essas, destaca-se o posicionamento baseado na viragem do $\mathrm{pH}$, defendida por alguns autores ${ }^{(12,23)}$.

Em tal modalidade de posicionamento, introduz-se o sensor de pH até a câmara gástrica e após deteç̧ão de pH ácido, traciona-se o mesmo lenta e gradualmente no sentido cranial, até identificar-se o ponto de viragem do $\mathrm{pH}$, caracterizado pelo ponto no qual há mudança do $\mathrm{pH}$ para níveis superiores a 4 . Considera-se que tal ponto coincide com a transição esôfago-gástrica e posiciona-se o sensor $5 \mathrm{~cm}$ acima desse local.

Há poucos trabalhos na literatura que comparam as duas modalidades de posicionamento do sensor (por manometria prévia e por viragem do $\mathrm{pH}$ ). Alguns autores, como KANTROWITZ et al. ${ }^{(1)}$, WALLTHER e DeMEESTER ${ }^{(27)}$, MARPLES et al. ${ }^{(15)}$, MATTOX e RICHTER ${ }^{(16)}$ e MATTOX et al. ${ }^{(17)}$ consideram que a identificação manométrica do EIE é imprescindível para o posicionamento adequado. Porém, outros, como ROKKAS et al. ${ }^{(22)}$, SCHINDLBECK et al. ${ }^{(23)}$, KLAUSER et al. ${ }^{(12)}$ e PEHL et al. ${ }^{(21)}$ advogam que o posicionamento baseado na viragem do pH é confiável e, em função disso, sugerem que não haveria a necessidade de submeter o paciente ao desconforto do estudo manométrico, para identificação do EIE.

É importante salientar que se o sensor de pHmetria for posicionado acima do local padronizado, pode-se subestimar a ocorrência de refluxo gastroesofágico (RGE). Por outro lado, se posicionado abaixo desse local, pode-se superestimar o refluxo e, em alguns casos, promover a detecção de pseudorefluxos.

Considerando a polêmica existente em relação à questão, o pequeno número de trabalhos prospectivos com amostra expressiva e metodologia adequada e o uso disseminado, em nosso meio, da técnica da viragem do $\mathrm{pH}$, idealizou-se o presente estudo.

\section{Objetivos}

Avaliar a adequação do posicionamento do sensor distal de $\mathrm{pHmetria}$ pela técnica de viragem do $\mathrm{pH}$, considerando-se a presença, o tipo e o grau de erro de posicionamento que tal técnica proporciona, tendo-se como referencial a localização padronizada, baseada na localização manométrica do EIE. Objetiva-se também estudar a influência, em tais parâmetros, da posição adotada pelo paciente durante a técnica da viragem.

\section{MÉTODOS}

Foram estudados de modo prospectivo, durante o período de 1 ano, pacientes encaminhados para realização de pHmetria esofágica prolongada, nos quatro Serviços participantes do presente trabalho: Laboratório de Investigação Funcional do Esôfago do Serviço de Cirurgia do Esôfago do Hospital das Clínicas da Faculdade de Medicina da Universidade de São Paulo; Setor de Motilidade Digestiva do Fleury: Medicina e Saúde, São Paulo, SP; Laboratório de Motilidade Digestiva da Clínica Cirúrgica do Aparelho Digestivo, São Paulo, SP e Laboratório de Motilidade Digestiva da Clínica Gastrovida, Curitiba, PR, doravante designados, respectivamente, por Serviços I, II, III e IV. O presente estudo foi aprovado nas Comissões de Ética em Pesquisa das instituições participantes. A aprovação da Instituição principal (CAPPesq HC-FMUSP) foi obtida em 17 de janeiro de 2007, sob o número 979/06.

No dia dos exames, os pacientes eram convidados a participar do estudo. Os que aceitaram, leram e assinaram termo de consentimento livre e informado. A seguir, eram registrados os dados demográficos e realizada entrevista clínica sistemática, com o objetivo de avaliar as queixas clínicas apresentadas.

Na seqüência, os pacientes foram submetidos a manometria esofágica, com equipamento Medtronic-Synetics e sonda com oito canais de registro, sob perfusão pneumohidráulica. Por tração lenta e gradual (meio a meio centímetro) da sonda, no sentido cranial, foi caracterizada a localização (distância em relação à narina) dos esfíncteres do esôfago. Foi anotada no protocolo de pesquisa a distância em centímetros da borda superior do EIE à narina.

Após identificação manométrica dos esfíncteres, os pacientes foram submetidos a pHmetria esofágica (com equipamento Medtronic-Synetics). Após introdução do sensor distal de $\mathrm{pH}$ na câmara gástrica (caracterizado pela identificação de $\mathrm{pH}$ menor que 4), o cateter era tracionado lenta (a cada 15 segundos) e gradualmente (de meio em meio centímetro) até que se identificasse o ponto de viragem de $\mathrm{pH}$, caracterizado como o ponto no qual há mudança do $\mathrm{pH}$ para valores superiores a 4 . A distância em centímetros desse ponto em relação à narina era anotada no protocolo de estudo.

Considerando-se que a distribuição do suco gástrico sofre influência gravitacional, realizou-se a identificação do ponto de viragem do $\mathrm{pH}$ de dois modos distintos, que caracterizaram dois grupos de estudo: com o paciente sentado (grupo I) e com o paciente em decúbito dorsal horizontal (grupo II). Após identificação e registro do ponto de viragem, o sensor distal do cateter de pHmetria era posicionado de modo a ficar na posição 
padrão, ou seja, $5 \mathrm{~cm}$ acima da borda superior do EIE, identificada pela manometria.

Registrou-se no protocolo de estudo o ponto onde seria posicionado o sensor distal de $\mathrm{pH}$, caso fosse adotada a técnica da viragem e que tipo de erro que tal procedimento acarretaria. Parte dos exames foi realizada com eletrodo com dois canais de registro. Para efeito do presente estudo, avaliou-se o posicionamento do sensor distal. Considerando-se erro, a diferença (em centímetros) entre a localização padronizada e a localização que seria adotada caso fosse empregada a técnica da viragem de $\mathrm{pH}$, conforme a seguinte "fórmula":

Erro $=$ diferença entre o posicionamento padronizado do sensor distal de $\mathrm{pHmetria}$ e o posicionamento que seria adotado se fosse empregada a técnica da viragem.

Em função de tal cálculo, considerou-se que:

- Diferença igual a zero: significa que não haveria erro (exemplo: 40,0 cm - 40,0 cm = zero);

- Diferença negativa: significa que haveria erro e que o sensor seria posicionado abaixo do local padronizado (exemplo: $40,0 \mathrm{~cm}-41,0 \mathrm{~cm}=-1 \mathrm{~cm}$ );

- Diferença positiva: significa que haveria erro e que o sensor seria posicionado acima do local padronizado (exemplo: $40,0 \mathrm{~cm}-38,0 \mathrm{~cm}=2 \mathrm{~cm})$.

Para efeito de caracterização do grau de erro, considerou-se que este seria grosseiro se fosse maior ou igual a $2 \mathrm{~cm}$. Foram incluídos no estudo todos pacientes que aceitaram participar e excluídos aqueles em que não se identificou acidificação intra-gástrica.

\section{Análise estatística}

Para a análise dos resultados foram utilizados o coeficiente de correlação de Pearson, o teste paramétrico $t$ de Student e os não-paramétricos: Mann-Whitney (para amostras independentes) e do qui ao quadrado para tabelas de contingência ${ }^{(24)}$. Em todos os testes fixou-se em 0,05 ou $5 \%$ o nível para rejeição da hipótese de nulidade.

\section{RESULTADOS}

Foram realizados no período do estudo 1.044 exames de pHmetria esofágica. Nove pacientes não aceitaram participar do trabalho; em quatro não foi possível detectar acidificação intra-gástrica. Desta forma, foram incluídos do estudo 1.031 pacientes. Conforme já destacado, foram classificados de acordo com a posição adotada durante a manobra de identificação da viragem do $\mathrm{pH}$, em dois grupos: grupo I (GI - 450 pacientes) e grupo II (GII - 581 pacientes).

Quanto às instituições onde se realizaram os exames, 141 $(13,7 \%)$ pacientes foram atendidos no Serviço I, $342(33,2 \%)$ no Serviço II, 449 (43,5\%) no Serviço III e 99 (9,6\%) pacientes no Serviço IV. Embora a participação de cada Serviço na composição da amostra tenha sido heterogênea, a distribuição entre os grupos de estudo em cada um deles foi bastante homogênea. Os exames realizados em São Paulo foram efetuados pelo autor principal deste trabalho e os realizados em Curitiba, por um dos co-autores. Contudo, destaca-se que o método de estudo empregado foi exatamente o mesmo. Não houve diferença significante quanto à distribuição dos pacientes nos grupos de estudo, nos quatro Serviços participantes $(P=0,343)$.

Em relação ao sexo, foram incluídos $588(57,0 \%)$ pacientes do sexo feminino e $443(43,0 \%)$ do masculino. Apesar de haver predomínio do sexo feminino nos dois grupos de estudo, não houve diferença significante entre eles, em relação à distribuição entre os $\operatorname{sexos}(P=0,198)$.

A idade variou de 12 a 86 anos (média de 44,2 anos). No GI, variou de 12 a 86 anos (média de 43,6 anos) e no GII de 12 a 84 anos (média de 44,7 anos). Não houve diferença significante quanto à idade dos pacientes nos dois grupos de estudo $(P=0,269)$.

Conforme aos sintomas clínicos, observou-se predomínio de queixas típicas em 512 pacientes $(49,7 \%)$ e de queixas atípicas e/ou extra-esofágicas em 519 (50,3\%). Salienta-se que, para os casos com predomínio de queixas atípicas e extra-esofágicas, recomenda-se $\mathrm{pHmetria}$ com dois sensores de $\mathrm{pH}$, que é impossível de ser realizada sem identificação manométrica do esfíncter superior do esôfago.

\section{Adequação do posicionamento do sensor distal pela técnica da viragem}

Se fosse adotada a técnica da viragem, haveria erro no posicionamento do sensor em 945 pacientes $(91,6 \%)$ : 411 (91,3\%) do GI e $534(91,9 \%)$ do GII. Portanto, ocorreria posicionamento na posição padronizada em apenas 86 pacientes $(8,4 \%): 39$ $(8,7 \%)$ do GI e $47(8,1 \%)$ do GII (Tabela 1$)$.

TABELA 1. Distribuição dos pacientes quanto à adequação do posicionamento do sensor de $\mathrm{pH}$ pela técnica da viragem, nos dois grupos de estudo

\begin{tabular}{lcccccc}
\hline \multirow{2}{*}{ Resultado } & \multicolumn{2}{c}{ Grupo I } & \multicolumn{2}{c}{ Grupo II } & \multicolumn{2}{c}{ Total } \\
\cline { 2 - 7 } & $\mathrm{n}$ & $\%$ & $\mathrm{n}$ & $\%$ & $\mathrm{n}$ & $\%$ \\
\hline Sem erro & 39 & 8,7 & 47 & 8,1 & 86 & 8,4 \\
Com erro & 411 & 91,3 & 534 & 91,9 & 945 & 91,6 \\
Total & 450 & 100,0 & 581 & 100,0 & 1031 & 100,0 \\
\hline$P=0,827$ (qui ao quadrado) & & & & &
\end{tabular}

Observa-se, contudo, que não houve diferença significante quanto ao erro nos dois grupos de estudo $(P=0,827)$, indicando que a posição adotada pelo paciente durante a técnica da viragem do $\mathrm{pH}$ não interfere no erro inerente à mesma.

Em relação à caracterização do grau de erro, observa-se que haveria erro considerado grosseiro em $597(63,2 \%)$ pacientes: $274(66,7 \%)$ do GI e $323(60,5 \%)$ do GII (Tabela 2).

Não houve diferença significante entre os dois grupos de estudo $(P=0,879)$, indicando que a posição adotada pelo paciente durante a técnica da viragem não interfere no grau de erro inerente às mesmas.

Em relação ao tipo de erro, nota-se que o sensor seria posicionado acima do local padronizado em $88(9,3 \%)$ pacientes [39 $(9,5 \%)$ do GI e em $49(9,2 \%)$ do GII] e seria posicionado abaixo do local padronizado em $857(90,7 \%)$ pacientes [372 (90,5 \%) do GI e em 485 (90,8 \%) do GII] (Tabela 3). 
TABELA 2. Distribuição dos pacientes quanto ao grau de erro nos dois grupos de estudo

\begin{tabular}{lccccccc}
\hline \multirow{2}{*}{ Grau de erro } & \multicolumn{2}{c}{ Grupo I } & \multicolumn{2}{c}{ Grupo II } & \multicolumn{2}{c}{ Total } \\
\cline { 2 - 8 } & $\mathrm{n}$ & $\%$ & $\mathrm{n}$ & $\%$ & $\mathrm{n}$ & $\%$ \\
\hline Erro menor que $2 \mathrm{~cm}$ & 137 & 33,3 & 211 & 39,5 & 348 & 36,8 \\
$\begin{array}{l}\text { Erro maior ou igual a } 2 \mathrm{~cm} \\
\text { grosseiro) }\end{array}$ & 274 & 66,7 & 323 & 60,5 & 597 & 63,2 \\
Total & 411 & 100,0 & 534 & 100,0 & 945 & 100,0 \\
\hline
\end{tabular}

$P=0,879$ (qui ao quadrado)

TABELA 3. Distribuição dos pacientes quanto ao tipo de erro (abaixo ou acima do local padronizado), nos dois grupos de estudo

\begin{tabular}{lcccccc}
\hline \multirow{2}{*}{ Tipo de erro } & \multicolumn{2}{c}{ Grupo I } & \multicolumn{2}{c}{ Grupo II } & \multicolumn{2}{c}{ Total } \\
\cline { 2 - 7 } & $\mathrm{n}$ & $\%$ & $\mathrm{n}$ & $\%$ & $\mathrm{n}$ & $\%$ \\
\hline Abaixo & 372 & 90,5 & 485 & 90,8 & 857 & 90,7 \\
Acima & 39 & 9,5 & 49 & 9,2 & 88 & 9,3 \\
Total & 411 & 100,0 & 534 & 100,0 & 945 & 100,0 \\
\hline
\end{tabular}

$P=0,959$ (qui ao quadrado)

Não houve diferença significante entre os dois grupos de estudo $(P=0,959)$, indicando que a posição adotada pelo paciente durante a técnica da viragem não interfere no tipo de erro inerente à mesma.

\section{DISCUSSÃO}

\section{Em relação ao desvio de posicionamento}

KANTROWITZ et al. ${ }^{(11)}$, comparando em 29 pacientes a localização da junção esôfago-gástrica, sugerida pela técnica da viragem do $\mathrm{pH}$, com a localização obtida por manometria esofágica, descrevem que a técnica da viragem não permitiu a identificação da junção em 7 casos $(24,1 \%)$. Em função disso, concluem que a manometria é necessária para o posicionamento adequado do sensor de pHmetria. Contudo, não fazem referência ao erro de posicionamento que a técnica da viragem propicia, como realizado no presente trabalho.

Por outro lado, KLAUSER et al.(12), comparando a localização do EIE obtida por manometria com a localização sugerida pela técnica da viragem do $\mathrm{pH}$ e pela fluoroscopia, em 60 pacientes com sintomas de DRGE e 14 voluntários assintomáticos, observaram que o ponto de viragem não ocorreu dentro dos limites do EIE em apenas 1 caso $(1,7 \%)$ e que a localização do esfíncter por fluoroscopia foi bastante falha. Esses autores, apesar de admitirem que a manometria esofágica é o método mais confiável para o posicionamento do sensor de pHmetria, concluem que a técnica da viragem do $\mathrm{pH}$ é eficaz na localização do EIE e questionam se 1 ou 2 centímetros de diferença no posicionamento do sensor teriam impacto relevante nos resultados do exame.
ROKKAS et al. ${ }^{(22)}$, em estudo comparativo da localização do EIE pela manometria esofágica com a técnica da viragem do $\mathrm{pH}$ em 46 pacientes com DRGE, referiram que a técnica da viragem identificou adequadamente a localização do esfíncter em $98 \%$ dos casos. Relataram que com o método obtêm-se diferença "aceitável" de 1 a $2 \mathrm{~cm}$ em relação à localização manométrica do esfíncter. Os autores concluem que a técnica da viragem representa alternativa confiável para o posicionamento do sensor de pHmetria.

A principal crítica ao método utilizado nas publicações de KLAUSER et al. ${ }^{(12)}$ e ROKKAS et al. ${ }^{(22)}$ é que os autores não compararam onde seria posicionado o sensor de $\mathrm{pHmetria}$, caso fosse adotada a técnica da viragem, como realizado no presente estudo. Convém salientar que, para o posicionamento adequado do sensor, precisa-se saber a localização da borda superior do esfíncter e não o intervalo no qual ele se localiza.

Conforme já salientado, não há consenso na literatura sobre a adequação do posicionamento do sensor de pHmetria pela técnica da viragem. Contudo, há de se destacar que os autores que consideram o método válido baseiam suas conclusões em trabalhos bastante criticáveis do ponto de vista metodológico e com amostras muito restritas.

A maioria desses autores considera toda a extensão do esfíncter e não somente a borda superior do mesmo. Ou seja, considera a viragem adequada se ocorrer na extensão de todo o esfíncter inferior, mas não fazem análise do grau de erro de posicionamento do sensor de $\mathrm{pH}$, que tal modalidade técnica proporciona.

O presente estudo permite concluir que o posicionamento do sensor distal de pHmetria pela técnica da viragem não é confiável, pois proporciona erro em $91,7 \%$ dos casos.

\section{Quanto ao grau do desvio}

PEHL et al. ${ }^{(21)}$ compararam a localização do EIE pela manometria esofágica com a técnica da viragem em 25 pacientes com sintomas de DRGE e 25 indivíduos controles assintomáticos. Simultaneamente, realizaram pHmetria com dois sensores de $\mathrm{pH}$ : um posicionado de acordo com a localização manométrica e outro de acordo com a técnica da viragem. Foram analisados os seguintes parâmetros: localização do EIE, número de episódios de refluxo, ocorrência de refluxo em posição ortostática e em decúbito horizontal e percentagem de tempo total de refluxo.

Os autores demonstraram que adotando a técnica da viragem, posicionariam o sensor de $\mathrm{pH}$, em média, $2 \mathrm{~cm}$ abaixo do local padronizado no grupo controle e $1 \mathrm{~cm}$ abaixo nos pacientes com DRGE. Em relação à mensuração do refluxo, observaram resultados semelhantes nos dois locais avaliados. Apesar de haver pequeno aumento do número de episódios de refluxo na posição ortostática no sensor posicionado de acordo com a técnica da viragem, não houve diferença significante em relação ao tempo total de refluxo.

Referem que em um paciente que estava em uso de antisecretores, não foi possível a realização da técnica da viragem e concluem que o cateter de pHmetria pode ser posicionado com segurança pela técnica de viragem. Porém, admitem que tal modalidade técnica é limitada em quem faz uso de anti- 
secretores e realiza a pHmetria para avaliação da eficácia do medicamento.

MATTOX et al. ${ }^{(17)}$ compararam a técnica da viragem com a manometria esofágica para localização do EIE em 71 pacientes com sintomas de DRGE e 14 voluntários assintomáticos, adotando os critérios de THURER et al. ${ }^{(26)}$, que admitem ser aceitável variação de $3 \mathrm{~cm}$ para cima ou $3 \mathrm{~cm}$ para baixo (total $6 \mathrm{~cm}$ ) da borda proximal do esfíncter.

Apesar de terem adotado margem de erro tão expressiva como aceitável, os autores demonstraram que a técnica da viragem identificou de modo inadequado o esfíncter em $58 \%$ dos pacientes com DRGE e em $29 \%$ dos voluntários assintomáticos, concluindo que a técnica não é eficiente para posicionamento do sensor de pHmetria.

WEUSTEN et al.$^{(28)}$ realizaram $\mathrm{pHmetria}$ com cinco sensores de registro, posicionados a 3, 6, 9, 12 e $15 \mathrm{~cm}$ acima da borda superior do EIE, em 19 pacientes com DRGE e em 19 controles assintomáticos. Observaram decréscimo gradual expressivo na detecção de refluxo da parte distal para a proximal do esôfago, tanto nos pacientes quanto nos controles assintomáticos. Tal achado representa crítica importante aos critérios de THURER et al. ${ }^{(26)}$.

No presente trabalho, viu-se que a técnica da viragem propicia erro no posicionamento do sensor de pHmetria em $91,7 \%$ dos casos e que em $63,2 \%$ das vezes tal erro é maior que $2 \mathrm{~cm}$. Em função dos dados apresentados, conclui-se que a técnica da viragem proporciona margem de erro expressiva.

\section{Quanto ao tipo de desvio}

Em relação ao tipo de erro, nota-se que o sensor seria posicionado abaixo do local padronizado em 857 (90,7\%) pacientes e acima dele em $88(9,3 \%)$.

Há na literatura apenas duas publicações que fazem referência específica a tal tema. PEHL et al. ${ }^{(21)}$, como já salientado, observaram que a técnica da viragem promove o posicionamento do sensor de $\mathrm{pH}$ em média $2 \mathrm{~cm}$ abaixo do local padronizado em controles assintomáticos e $1 \mathrm{~cm}$ abaixo nos pacientes com DRGE. WALLTHER e DeMEESTER ${ }^{(27)}$ também demonstraram que a técnica da viragem do $\mathrm{pH}$ tende a propiciar o posicionamento do sensor de pHmetria abaixo do local padronizado.

Em função desses dados, é interessante avaliar o impacto na mensuração do refluxo que diferentes grandezas de erro, efetivamente, propiciam.

LEHMAN et al. ${ }^{(13,14)}$, comparando o número de episódios de refluxo registrados em sensores posicionados a 1 e $5 \mathrm{~cm}$ acima do EIE, observaram que a detecção de refluxo no sensor distal era três vezes maior que a detecção na posição padrão. DICKMAN et al. ${ }^{(5)}$, comparando o percentual de tempo de refluxo registrado em sensores posicionados a 1 e $6 \mathrm{~cm}$ acima do EIE, em 64 pacientes com sintomas de DRGE, também constataram que o percentual de refluxo era expressivamente maior a $1 \mathrm{~cm}$.

FLETCHER et al. ${ }^{(6)}$ compararam o percentual de tempo de refluxo registrado em sensores posicionados a 0,5 e $5,5 \mathrm{~cm}$ acima da junção escamocolunar em 11 pacientes dispépticos com pHmetria convencional normal. Observaram que o percentual médio de tempo total de refluxo foi 11,7 no sensor distal e 1,8 no proximal. Diferentemente dos dois estudos anteriormente citados, os autores fixaram por meio de presilhas metálicas o sensor distal à mucosa esofágica, eliminando a possibilidade de detecção de pseudorefluxos.

ANGGIANSAH et al. ${ }^{(2)}$, comparando o número de episódios de refluxo detectados em sensores localizados a 5 e $10 \mathrm{~cm}$ acima do EIE, constataram que havia detecção expressivamente menor de refluxo no sensor posicionado acima da localização padronizada. JOHANSSON e TIBBLING ${ }^{(8)}$, comparando o número de episódios de refluxo detectados em sensores posicionados a 5 e $15 \mathrm{~cm}$ acima do EIE em 39 pacientes com DRGE, também constataram menor detecção de refluxo no sensor proximal.

Parece claro que a monitorização do $\mathrm{pH}$ realizada com sensores posicionados acima da localização padronizada proporciona menor detecção de refluxo. Por outro lado, a monitorização com sensores mais distais proporciona maior detecção. Em função disso, é inevitável a questão: por que monitorizar o refluxo a $5 \mathrm{~cm}$ acima do EIE se podemos detectar mais refluxo monitorizando um pouco mais abaixo?

SIFRIM et al. ${ }^{(25)}$ demonstraram que há encurtamento do esôfago durante as deglutições. Dessa forma, se se posicionar o sensor de $\mathrm{pH}$ muito próximo da transição, pode haver exposição do sensor ao conteúdo intragástrico e a caracterização de falsos episódios de refluxo. Em função disso, acredita-se que estudos que se proponham a fazer a monitorização bastante distal do refluxo devem ser feitos com sensores fixados à mucosa esofágica, para impedir o fenômeno descrito.

Essa seria uma das principais vantagens da $\mathrm{pHmetria} \mathrm{esofágica}$ sem cateter ou seja, com a monitorização do $\mathrm{pH}$ intra-esofágico por meio de cápsulas fixadas à mucosa esofágica, que transmitem os dados captados por sinal de rádio. Com tal método, pode-se realizar a monitorização mais distal do refluxo evitando-se a detecção de pseudorefluxos.

Os resultados encontrados no presente trabalho indicam que a técnica da viragem não representa boa opção para o posicionamento do sensor de pHmetria. Entretanto, como já salientado, há outra grande questão a ser respondida: será que o local padronizado de monitorização é o mais adequado para análise do refluxo?

\section{Influência da posição do paciente durante a manobra da viragem do $\mathrm{pH}$}

Pelo presente estudo, avaliando-se: erro de posicionamento, margem de erro e tipo de erro que a técnica da viragem do $\mathrm{pH}$ proporciona, nas posições sentado e deitado, constatou-se que não há influência na posição adotada pelo paciente durante a manobra da viragem, em nenhum dos parâmetros analisados.

WALLTHER e DeMEESTER ${ }^{(27)}$ compararam a localização da borda superior do EIE por endoscopia e pela técnica de viragem e a influência da posição adotada pelo paciente durante a manobra da viragem. Adotaram a localização manométrica do esfíncter como referencial, em modelo de estudo semelhante ao presente, porém, com número muito restrito de casos (20 pacientes). Os autores observaram que a localização do esfíncter pela técnica da viragem foi mais precisa que localização endoscópica. Contudo, destacam que apesar da superioridade da técnica da viragem, a mesma identificou corretamente (com margem de erro aceitável de $1 \mathrm{~cm}$ ) a borda superior do EIE em apenas quatro $(20,0 \%)$ pacientes. De modo semelhante ao 
observado no presente trabalho, demonstraram que a técnica da viragem do $\mathrm{pH}$ tende a propiciar o posicionamento do sensor de pHmetria abaixo do local padronizado. Concluem que a técnica da viragem não é suficientemente precisa para ser empregada na prática clínica e que a posição adotada pelo paciente durante a manobra não interfere na margem de erro inerente à técnica.

Não foram encontrados na literatura outros estudos sobre a influência da posição do paciente na técnica da viragem.

Com o presente estudo, deu-se boa contribuição ao estudo da modalidade alternativa de posicionamento do sensor de $\mathrm{pHmetria}$ mais utilizada - a técnica da viragem do $\mathrm{pH}$. Viu-se que a mesma proporciona erro expressivo de posicionamento, tendendo a posicionar o sensor em local mais distal que o padronizado.

\section{CONCLUSÕES}

Nas condições da presente pesquisa, pode-se concluir que:

1. O posicionamento do sensor distal de $\mathrm{pHmetria}$ pela técnica da viragem do $\mathrm{pH}$ não é confiável.

2. A técnica da viragem proporciona margem de erro expressiva.

3. O tipo de erro mais comum que tal modalidade técnica proporciona é o posicionamento mais distal do sensor, que pode superestimar a ocorrência de refluxo e, em alguns casos, promover a detecção de pseudorefluxos.

4. Não há influência da posição adotada pelo paciente durante a realização da técnica da viragem do $\mathrm{pH}$, na eficiência do método.

Nasi A, Frare RC, Brandão JF, Falcão AM, Michelsohn NH, Sifrim D. Comparative prospective study of two positioning modes of 24-hour esophageal pH monitoring: by esophageal manometry and by the $\mathrm{pH}$ step-up technique. Arq Gastroenterol. 2008;45(4):261-7.

ABSTRACT - Background - By internationally accepted standardization, the esophageal pH-meter distal sensor is positioned $5 \mathrm{~cm}$ above the superior border of the lower esophageal sphincter, identified by esophageal manometry. However, several authors suggest alternative positioning techniques that leave out the manometry; among such techniques, the $\mathrm{pH}$ step-up is the one to be pointed out. This subject is controversial; some publications state that the step-up technique is not reliable while some others consider it reliable. Aims - Considering the existent controversy and the small number of prospective works with suitable sample and methodology, we have idealized the present study, that aims the evaluation of the suitability of the pH-meter distal sensor positioning based on the step-up technique, by analyzing the presence, the type and the degree of the error of positioning that such technique provides and the influence of the position adopted by the patient during the procedure. Methods - One thousand and thirty one patients conducted to the esophageal $\mathrm{pH}$-meter procedure were studied in a prospective way. During the clinical interview, the demographic data and the presented clinical complaints were registered. All the patients were submitted to both esophageal manometry in order to localize the lower esophageal sphincter and the $\mathrm{pH}$ step-up technique, that consists of the introduction of the $\mathrm{pH}$-meter sensor in the gastric chamber and in the sensor's gradual traction until the $\mathrm{pH}$ steps up to levels over 4 . The step-up point was identified by two distinct ways, characterizing two study groups: with the sitting patient (group I - 450 patients) and with the patient in supine position (group II - 581 patients). After the step-up point identification, the $\mathrm{pH}$-meter distal sensor was placed in the standard position (based on the sphincter manometric placement). It was registered where the $\mathrm{pH}$ sensor would be positioned if the step-up technique were adopted. To evaluate the positioning suitability, the error was considered to be represented by the difference (in centimeters) between the suitable placement (manometry) and the one that would be adopted in case the step-up technique were adopted. The positioning error was considered rough if it were larger than $2 \mathrm{~cm}$. The most frequent type of error was also analyzed (if above or below the standard position). Results: It was observed that if the step-up technique were adopted, there would be error in the sensor positioning in 945 patients $(91.6 \%)$. In terms of error degree, there would be a rough error in 597 (63.2\%) cases. Concerning the type of error, the sensor would be positioned below the standard place in 857 (90.7\%) patients. As to the interference of the position adopted by the patient during the step-up technique, it was observed that there was no significant difference among the groups of study in any of the analyzed parameters. Conclusions - 1. The positioning of the pH-meter distal sensor by the step-up technique is not reliable; 2. the step-up technique provides expressive error margin; 3 . the most common type of error that such technique mode provides is the placement of the sensor below the standard positioning, which may overestimate the reflux occurrence; 4. there is no influence in the position adopted by the patient during the $\mathrm{pH}$ step-up technique procedure, in terms of method efficiency.

HEADINGS - Esophageal pH monitoring. Gastroesophageal reflux, diagnosis. Esophagitis, peptic, diagnosis. Esophageal sphincter, lower. Comparative study. 


\section{REFERÊNCIAS}

1. American Gastroenterological Association. Clinical esophageal $\mathrm{pH}$ recording: a technical review for practice guideline development. Gastroenterology. 1996;110:1982-96.

2. Anggiansah A, Sumboonnanonda K, Wang J. Significantly reduced acid detection at 10 centimeters compared to 5 centimeters above lower esophageal sphincter in patients with acid reflux. Am J Gastroenterol. 1993;88:842-6.

3. DeMeester TR, Wang C, Wernly JA, Pellegrini CA. Technique, indications, and clinical use of 24 hour esophageal pH monitoring. J Thorac Cardiovasc Surg. 1980;79: 656-70.

4. DeVault KR, Castell DO. Updated guidelines for the diagnosis and treatment of gastroesophageal reflux disease. Am J Gastroenterol. 1999;6:1434-42.

5. Dickman R, Bautista JM, Wong WM, Bhatt R, Beeler JN, Malagon I, Risner-Adle S, Lam KF, Fass R. Comparison of esophageal acid exposure distribution along the esophagus among different gastroesophageal reflux disease groups. Am J Gastroenterol. 2006;101:2463-9.

6. Fletcher J, Wirz A, Henry E, McColl KEL. Studies of acid exposure immediately above the gastro-oesophageal squamocolumnar junction: evidence of short segment reflux. Gut. 2004;53:168-73

7. Jamieson JR, Stein HJ, DeMeester TR, Bonavina L, Schwizer W, Hinder RA, Albertucc M. Ambulatory $\mathrm{pH}$ monitoring: normal values, optimal thresholds, specificity, sensitivity, and reproducibility. Am J Gastroenterol. 1992;87:1102-11.

8. Johansson KE, Tibbling L. Gastric secretion and reflux pattern in reflux oesophagitis before and during ranitidine treatment. Scand J Gastroenterol. 1986;21:487-92.

9. Johnson LF, DeMeester TR. Twenty-four-hour $\mathrm{pH}$ monitoring of the distal esophagus: a quantitative measure of gastroesophageal reflux. Am J Gastroenterol. 1974;62: 325-32.

10. Kahrilas PJ, Quigley EMM. American Gastroenterological Association medical position statement: guidelines on the use of esophageal $\mathrm{pH}$ recording. Gastroenterology. 1996;110:1981-96.

11. Kantrowitz PA, Corson JG, Fleischli DJ, Skinner DB. Measurement of gastroesophageal reflux. Gastroenterology. 1969;56:666-74.

12. Klauser AG, Schindlbeck NE, Muller-Lissner SA. Esophageal 24-h pH monitoring: is prior manometry necessary for correct positioning of the eletrode? Am J Gastroenterol. 1990;85:1463-7.

13. Lehman G, O'Connor K, Cravens E, Flueckiger J, Kopecky K. Does placement of $\mathrm{pH}$ probes less than $5 \mathrm{~cm}$ above the lower esophageal sphincter (LES) produce falsely positive gastroesophageal reflux? [abstract]. Gastroenterology. 1988;94:A255.

14. Lehman G, Rogers D, Cravens E, Flueckiger J. Prolonged $\mathrm{pH}$ probe testing less than $5 \mathrm{~cm}$ above the lower esophageal sphincter (LES): establishing normal control values [abstract]. Gastroenterology. 1990;98:A77.
15. Marples MI, Mughal M, Bancewicz J. Can a esophageal $\mathrm{pH}$ electrode be accurately positioned without manometry? In: Siewert JR, Holscher AH, editors. Diseases of the esophagus. Berlin: Springer-Verlag; 1987. p.789-91.

16. Mattox HE III, Richter JE, Sinclair JW, Prince JE, Case LD. Gastroesophageal pH step-up inaccurately locates proximal border of lower esophageal sphincter. Dig Dis Sci. 1992;37:1185-91.

17. Mattox HE III, Richter JE. Prolonged ambulatory esophageal $\mathrm{pH}$ monitoring in the evaluation of gastroesophageal reflux disease. Am J Med. 1990;89:345-55.

18. Moraes-Filho JPP, Cecconello, IC, Gama-Rodrigues J, Castro LP, Henry MA, Meneghelli UG, Quigley E, Brazilian Consensus Group. Brazilian consensus on gastroesophagea reflux disease: proposals for assessment, classification, and management. Am J Gastroenterol. 2002;97:241-8.

19. Nasi A, Moraes-Filho JPP, Zilberstein B, Cecconello I, Gama-Rodrigues JJ, Pinotti HW Gastroesophageal reflux disease: clinical, endoscopic and intraluminal esophageal $\mathrm{pH}$ monitoring evaluation. Dis Esophagus. 2001;14:41-9.

20. Nasi A, Moraes-Filho JPP, Cecconello I. Gastroesophageal reflux disease: an overview. Arq Gastroenterol. 2006;43:334-41.

21. Pehl C, Boccali I, Hennig M, Schepp W. pH probe positioning for 24-hour $\mathrm{pH}$-metry by manometry or pH step-up. Eur J Gastroenterol Hepatol. 2004;16:375-82.

22. Rokkas TH, Anggiansah A, Dorrington L, Owen WJ, Sladen GE. Accurate positioning of the pH-probe in the oesophagus without manometry. Ital J Gastroenterol. 1987; $19: 176-8$

23. Schindlbeck NE, Heinrich C, Konig A, Dendorfer A, Pace F, Muller-Lissner SA Optimal thresholds, sensitivity, and specificity of long-term $\mathrm{pH}$-metry for the detection of gastroesophageal reflux disease. Gastroenterology. 1987;93:85-90.

24. Siegel S. Estatística no paramétrica. México: Trilhas; 1975. 364p.

25. Sifrim D, Tack J, Janssens J. Ambulatory continuous monitoring of esophageal shortening in man. Preliminary observations in patients with chest pain. Gastroenterology. 2003;124(Suppl 1):a121-2.

26. Thurer RL, DeMeester TR, Johnson LF. The distal esophageal sphincter and its relationship to gastroesophageal reflux. J Surg Res. 1974; 16:418-23.

27. Wallther B, DeMeester TR. Placement of the esophageal $\mathrm{pH}$ electrode for 24-hou oephageal pH monitoring. In: DeMeester TR, Skinner BD, editors. Esophageal disorders: pathophysiology and therapy. New York: Raven Press; 1985. p.539-41.

28. Weusten BLAM, Akkermans LMA, Vanberge-Henegouwen GP, Smout AJPM. Dynamic characteristics of gastro-oesophageal reflux in ambulatory patients with gastro-oesophageal reflux disease and normal control subjects. Scand J Gastroenterol 1995;30:731-7.

Recebido em 26/10/2007. Aprovado em 11/1/2008 